\title{
Incidence of Antibiotic Resistance Bacteria in Jeddah's Ministry of Health Hospitals, Saudi Arabia
}

\author{
Muhammad A. Halwani' ${ }^{1}$, Nidal A. J. Tashkandy², Magda M. Aly3, Saad B. Al Masoudi³, \\ Osama 0. Dhafar ${ }^{2}$ \\ ${ }^{1}$ Department of Microbiology, Faculty of Medicine, Al Baha University, Al Baha, Saudi Arabia \\ ${ }^{2}$ Health Affairs, Jeddah, Saudi Arabia \\ ${ }^{3}$ Biology Department, Faculty of Science, King Abdulaziz University, Jeddah, Saudi Arabia \\ Email: *magdammali@hotmail.com
}

Received 8 October 2015; accepted 13 November 2015; published 16 November 2015

Copyright (C) 2015 by authors and Scientific Research Publishing Inc.

This work is licensed under the Creative Commons Attribution International License (CC BY). http://creativecommons.org/licenses/by/4.0/

(c) (i) Open Access

\section{Abstract}

This study aimed to determine the emergence and spread of resistant bacteria in Jeddah Ministry of Health hospitals. Sixteen month follow-up (January 2010 to April 2011) study was carried out and clinical isolates of hospitalized patients were collected, identified and their antimicrobial resistance was determined using two automated systems, Phoenix and Vitek 2. Results revealed that 6195 isolates were identified of which 94\% (5846/6195) were Gram negatives. In Escherichia coli, the resistance was $40 \%(681 / 1703)$ to ciprofloxacin, $30 \%(511 / 1703)$ to cefepime, $29 \%(494 / 1703)$ to ceftazidime, $8.5 \%(145 / 1703)$ to tazocin and amikacin, $40 \%(681 / 1703)$ to gentamicin and cefuroxime. In Klebsiella pneumonia, the resistance was $48 \%(550 / 1147)$ to ceftazidime, $49 \%$ $(565 / 1147)$ to cefuroxime, $45.5 \%(522 / 1147)$ to cefepime, $38 \%(436 / 1147)$ to gentamicin, $30 \%$ (344/1147) to ciprofloxacin, $19 \%(218 / 1147)$ to tazocin, $7.5 \%(86 / 1147)$ to amikacin and $2.4 \%$ (27/1147) to imipenem/meropenem. In Acinetobacter bumannii, 79\% (850/1076) were resistant to ciprofloxacin, $68.5 \%(737 / 1076)$ to tazocin, $67 \%(721 / 1076)$ to cefepime, $66 \%(710 / 1076)$ to gentamicin and imipenem/meropenem, 65\% (699/1076) to ceftazidime, $68 \%(735 / 1076)$ to amikacin and no resistance to colistin was reported. In Pseudomonas aeruginosa, almost 34\% (555/1632) were resistant to ceftazidime, $31 \%(506 / 1632)$ to ciprofloxacin, $29 \%(473 / 1632)$ to cefepime, $26.5 \%(434 / 1638)$ to gentamicin, $19 \%(310 / 1632)$ to imipenem/meropenem, $17 \%$ $(277 / 1632)$ to amikacin, and $15.5 \%(253 / 1632)$ were resistant to tazocin. In Gram positive isolates, MRSA counted only for $4.6 \%(302 / 6552)$ and no vancomycin intermediate Staphylococcus aureus (VISA) were detected. In conclusion, the resistance detected in this study is considered high and antibiotic Stewardship Programs is inevitably required.

\footnotetext{
"Corresponding author.
}

How to cite this paper: Halwani, M.A., Tashkandy, N.A.J., Aly, M.M., Al Masoudi, S.B. and Dhafar, O.O. (2015) Incidence of Antibiotic Resistance Bacteria in Jeddah's Ministry of Health Hospitals, Saudi Arabia. Advances in Microbiology, 5, $780-786$. http://dx.doi.org/10.4236/aim.2015.512082 


\section{Keywords}

\section{Resistance, Clinical Isolates, Jeddah, Klebsiella, Antibiotic}

\section{Introduction}

In Kingdom of Saudi Arabia, Jeddah is the second largest city with commercial importance and with a population of 3.2 million people. It is the gateway to the holy cities of Makkah and Medina [1]. The Ministry of Health oversees Jeddah's health system and provides free medical care to Saudi citizens and legal residents. The Ministry of Health in Saudi Arabia is a major source of medical services in the country and has 12 different hospitals distributed among the city with approximately a total of 2400 beds. The infection prevention and control program in those hospitals is covered by one specialized administration that runs a scientifically based standardized program. All microbiology results and antibiotic susceptibilities are sent to the administration on a monthly basis for follow-up and analysis. Data are checked for validly and accuracy with the microbiologists in the hospitals.

Antimicrobial drugs decreased death and illness associated with infectious diseases in however there is an emergence and spread of drug-resistance isolates among bacteria [2]. Antibacterial resistant isolates caused outbreaks, and more involved in health care-associated infections: including bacteremia, pneumonia, meningitis, urinary tract infection, and wound infection and greatly limited the therapeutic options for infected patients [3]. Moreover, the developed resistance for a major class of antimicrobial drugs appeared as short as 1 year to $>10$ years [4]. What's more, there is a major increase in the emergence and spread of multidrug-resistant bacteria to newer compounds, such as cephalosporins and fluoroquinolones [5].

The more problematic drug-resistant pathogens include multidrug-resistant Acinetobacter baumannii, Klebsiella pneumoniae, Escherichia coli, and Pseudomonas aeruginosa among the gram-negative bacteria and methicillin-resistant Staphylococcus aureus, penicillin-resistant Streptococcus pneumoniae, and vancomycin-resistant enterococci among the gram-positive bacteria [6].

Some E. coli isolates caused urinary tract infection, meningitis, peritonitis intestinal and extra-intestinal infections and their resistance for the longest used antimicrobial agents was consistently the highest [7]. During a 12-year period (1971-1982), the susceptibility of $E$. coli, isolated from hospitals, showed no major change in resistance to all tested antimicrobial drugs [7]. In contrast, E. coli collected during 1997-2007 from urine specimens showed increasing resistance to ciprofloxacin, trimethoprim/sulfamethoxazole, and amoxicillin/clavulanic acid [8]. In a 30-year (1979-2009) follow-up study in Sweden, E. coli showed an increasing resistance towards ampicillin, sulfonamide, trimethoprim, and gentamicin [9] and this yet was a great concern.

In intensive care units, Klebsiella spp. is among the most common isolated pathogens and the emergence of $K$. pneumoniae resistance to antibiotics is well documented [10]. The study of Sanchez et al. [11] showed that $K$. pneumoniae antimicrobial drug resistance increased for every antimicrobial class studied except tetracyclines and cross-resistance among imipenem-resistant $K$. pneumoniae was high for ciprofloxacin but lower for amikacin and tetracycline.

Pseudomonas aeruginosa, associated with nosocomial infections, rapidly developed resistance to multiple classes of antibiotics and the import of resistance mechanisms on mobile genetic elements was always a concern [12]. Multi-drug-resistant $P$. aeruginosa was intermediate or resistant to at least three drug classes: $\beta$-lactams, carbapenems, aminoglycosides, and fluoroquinolones and the reported rates varied from $0.6 \%-32 \%$ based on geographic location and the type of surveillance study [13]. Increasing emergence and spread of resistant Acinetobacter strains to the first and second generation cephalosporins started since 1975 but during 1980s to 1990s, the worldwide spread of imipenem resistant strains was recorded that could combat many severe Acinetobacter infections. Furthermore, carbapenem resistance in the strains of A. baumannii decreased the therapeutic options and made the life of treating physicians and patients really miserable [14]. The aim of the study was to detect the incidence of resistance in clinical bacterial isolates that was identified in 12 hospitals at Jeddah.

\section{Material \& Methods}

\section{Bacterial Identification and Sensitivity}

The study was conducted for 16 months (January 2010 to April 2011) in 12 Ministry of Health hospitals in Jed- 
dah, Saudi Arabia. During that period, 6195 non-repetitive clinical isolates from various specimens of hospitalized patients were collected and tested against the routinely used antibiotics. Two main automated systems are available and used in Jeddah hospitals laboratories; Phoenix (Becton Dickinson Diagnostic Systems, Sparks, MD, USA) and Vitek 2 (bioMérieux, Marcy l'Étoile, France). Susceptibilities of the Gram negative isolates were tested to the following antibacterial agents: Ceftazidime, Ciprofloxacin, Cefepime, Amikacin, Tazocin, Gentamicin, Cefuroxime and Imipenem. Colistin was tested only when an isolate showed a complete pattern of resistance to all tested antibiotics. All bacterial isolates were tested using standard bacteriological procedures and according to the procedures recommended by the manufacturers. No attempt was made to compare between the results of the two methods in this study.

\section{Results}

Sixteen month follow-up (January 2010 to April 2011) of the clinical isolates, obtained from hospitalized patients, in the 12 Ministry of Health hospitals was carried out. The counts and resistance of some of the Gram positive and negative bacterial isolates were identified using in-house diagnostic microbiology methods. Figure 1 revealed that 6195 isolates were identified of which 94\% (5846/6195) were Gram negatives and 6\% (637/6195) were Gram positive. Furthermore, E. coli was the most isolated organism and counted for $27 \%(1703 / 6195)$ of all identified isolates followed by P. aerignosa 26\% (1632/6195), K. pneumoniae 18\% (1147/6195), Acinetobacter bumannii 17\% (1076/6195) and Enterobacter cloacae 4.6\% (288/6195). MRSA counted for almost 5\% (349/6195) and Streptococcus pneumoniae for 0.7\% (47/6195). The rate of resistance in Gram negative isolates is shown in Figures 2-5.

In Escherichia coli; 40\% (681/1703) were resistance to ciprofloxacin, gentamicin or cefuroxime, 30\% (511/1703) were resistant to cefepime, 29\% (494/1703) to ceftazidime, $8.5 \%(145 / 1703)$ to tazocin and amikacin, $40 \%$ (681/1703) to (Figure 2). In Klebsiella pneumonia isolates, 48\% (550/1147) were resistant to ceftazidime, $49 \%$ (565/1147) to cefuroxime, $45.5 \%$ (522/1147) to cefepime, 38\% (436/1147) to gentamicin, 30\% (344/1147) to ciprofloxacin, $19 \%(218 / 1147)$ to tazocin, $7.5 \%$ (86/1147) to amikacin and $2.4 \%$ (27/1147) to imipenem/meropenem (Figure 3). In A. bumannii, 79\% (850/1076) were resistant to ciprofloxacin, 68.5\% (737/1076) to tazocin, 67\% (721/1076) to cefepime, 66\% (710/1076) to gentamicin and imipenem/meropenem, 65\% (699/1076) to ceftazidime, 68\% (735/1076) to amikacin and no resistance to colistin was reported (Figure 4). In Pseudomonas aeruginosa, almost 34\% (555/1632) were resistant to ceftazidime, 31\% (506/1632) to ciprofloxacin, 29\% (473/1632) to cefepime, $26.5 \%$ (434/1638) to gentamicin, 19\% (310/1632) to imipenem/meropenem, $17 \%$ (277/1632) to amikacin, and 15.5\% (253/1632) were resistant to tazocin (Figure 5).

In Gram positive isolates; out of 357 Staphylococcus aureus isolates collected, 302 (89\%) were MRSA which counted only for $4.6 \%(302 / 6552)$ of the total collected isolates. No vancomycin intermediate Staphylococcus

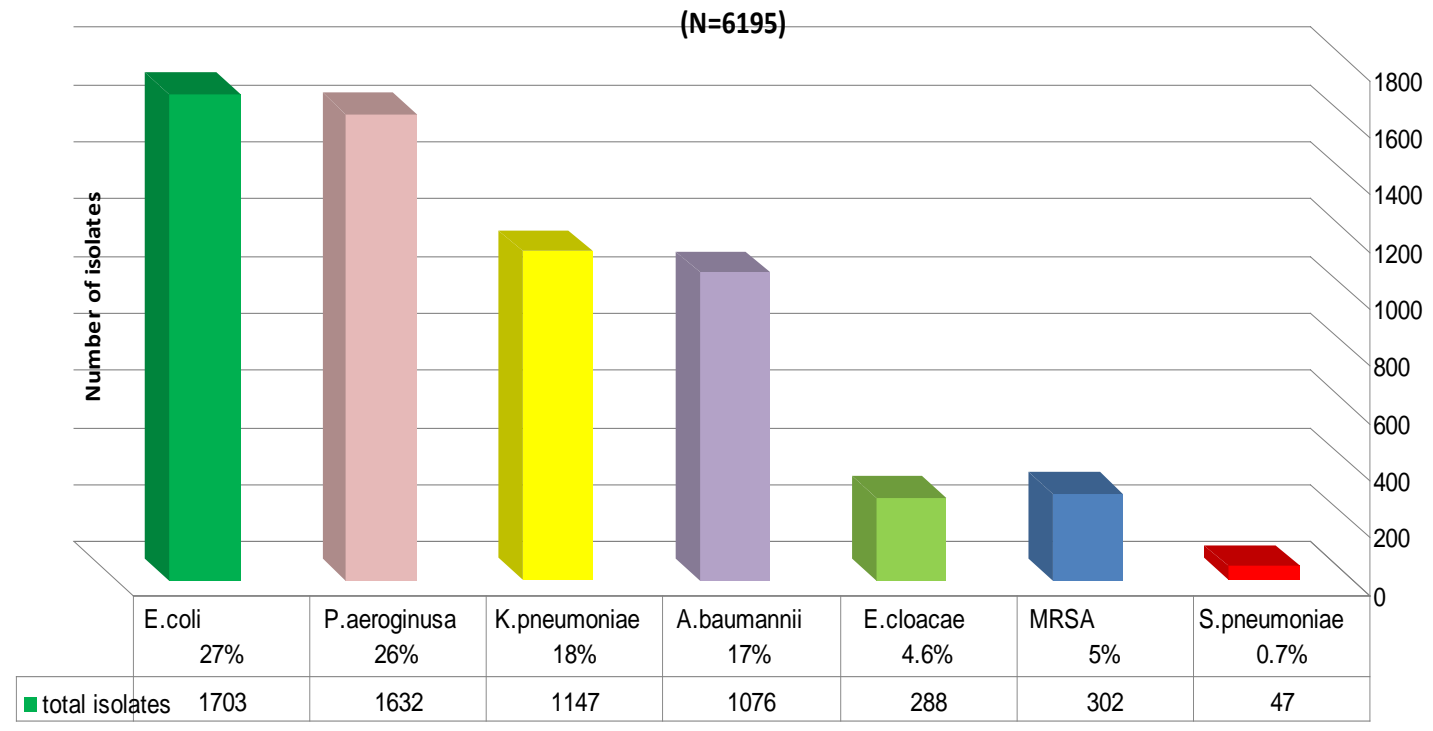

Figure 1. The different pathogenic bacterial isolates and their rates. 

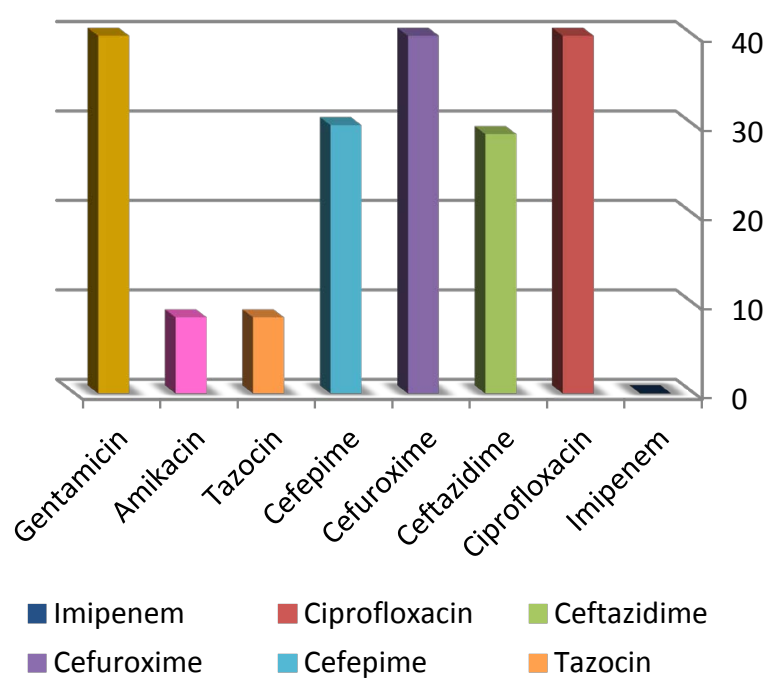

Figure 2. The overall resistance rate of $E$. coli isolates $(\mathrm{N}=$ 1703) to different used antibiotics.
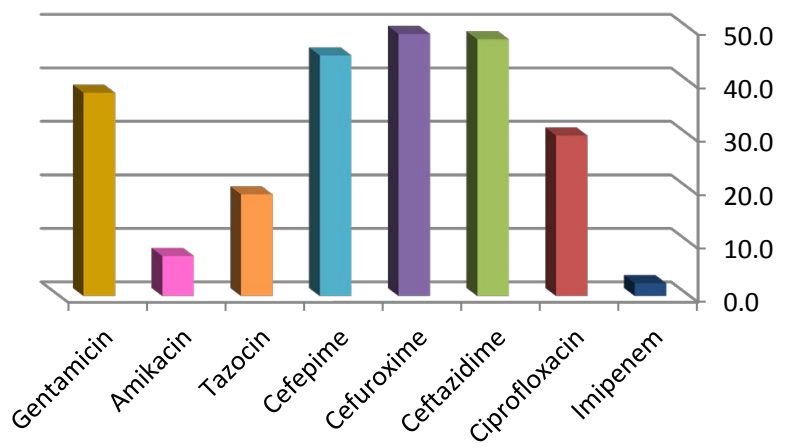

$\begin{array}{lll}\square \text { Imipenem } & \text { Ciprofloxacin } \\ \text { Cefepime } & \text { Ceftazidime } & \text { Cefuroxime } \\ \text { Tazocin } & \text { Amikacin }\end{array}$

Figure 3. The overall resistance rate of $K$. pnermoniae isolates $(\mathrm{N}=1147)$ to different used antibiotics.
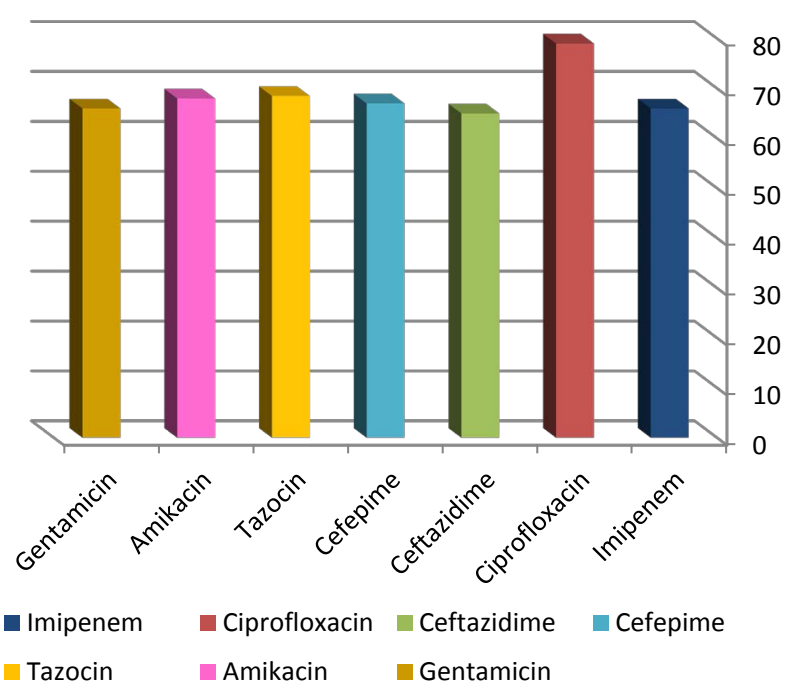

Figure 4. The overall resistance rate in A. bumannii isolates (N $=1076$ ) to different used antibiotics. 


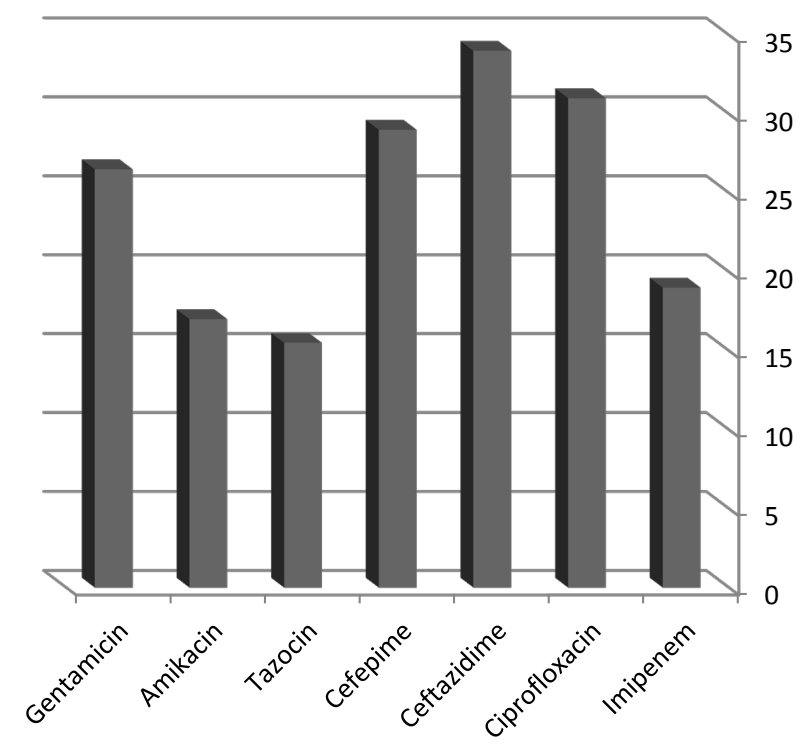

Figure 5. The overall resistance rate in $P$. aeruginosa isolates $(\mathrm{N}=1632)$ to different used antibiotics.

aureus (VISA) were detected. Furthermore, only 10\% (5/47) Streptococcus pneumonia isolates were resistant to penicillin (data not shown).

\section{Discussion}

Bacterial pathogens have a great ability to adapt and overcome the challenges of antibiotics in their environment that threaten to move us into the "post-antibiotic era" of infectious diseases. The therapy for resistant bacterial infections becomes more problematic than ever and the infection prevention is now very essential [15] [16]. Our study has some limitations where identification of the multi-drug resistance isolates is performed with two different automated systems and this may have given slight variations of the antibiotics' break points. Hence, standardization of lab identification methodologies in all hospital labs might be desirable.

Our study revealed E. coli is the dominating organism (27\%) in comparison to other Gram negatives bacteria. In addition, $P$. aeruginosa was found to be the second isolated organism (26\%) which might highlight the heavy involvement of hospital environment. Lower percentages $18 \%$ and $17 \%$ were recorded in K. pneumoniae and $A$. baumonnii, respectively. In a study that was conducted by Wagenlehner et al. [17], E. coli counted for 50\% of the yielded isolates, Proteus spp. 15\%, Klebsiella spp. 15\% and P. aeruginosa 5\%. The resistance rates reported in this study was disturbing to ciprofloxacin, gentamicin or cefuroxime (40\%) in E. coli, to cefuroxime (49\%) and ceftazidime (48\%) in K. pneumonia. Similarly, Livermore et al. [18] reported the changes in susceptibility pattern of E. coli and during 2011 in Central Greece, a significant increase in E. coli ciprofloxacin resistance (21\%) has occurred [19]. The emergence of extended resistant Acinetobacter spp. was rapidly spreading to even newer antimicrobials and they acquired resistance faster than other Gram-negative organisms due to their ease of survival in the hospital environment, their immense potential to cause nosocomial outbreaks and their biofilm forming ability which played a crucial role in their in-vitro and in-vivo survival [20]. This study showed that $\geq 66 \%$ of $A$. bumannii isolates were resistant to almost all tested antibiotics and this clearly indicated how problematic to have patients infected with this organism and the minimal chances available to find proper treatment.

Increasing resistance of $P$. aeruginosa to the various antipseudomonal agents has been reported worldwide which cause a serious problem in infection management. Although up to $34 \%$ of $P$. aeruginosa in our study isolates were resistant to ceftazidime and $31 \%$ to Ciprofloxacin, other alternative antibiotics are available. This high resistance clearly indicates the overuse of the latter antibiotics and insists the demand of a strictly respected comprehensive antibiotic stewardship program in our hospitals. In United States, a dramatic antimicrobial resistance increase to ciprofloxacin (from 15\% to 32\%) and ceftazidime (from 15\% to 19\%) over the ten-year period was recorded [21]. In Kuala Lumpur, Malaysia, approximately 10\% of clinical isolates of P. aeruginosa were resistant to imipenem, while $11 \%$ showed resistance to ciprofloxacin, piperacillin and ceftazidime [22] [23]. 
Despite the increasing resistance to ceftazidime, it was still in use against $P$. aeruginosa infections as the resistance did not exceed $35 \%$ in our study.

Staphylococcus aureus cause health care-associated infections for both hospitalized patients with healthy or decreased host defenses. In the 1980s, methicillin-resistant S. aureus (MRSA) emerged as a major clinical and epidemiologic problem in hospitals and spreaded out of the hospitals into communities. In the present study, MRSA counted only for $4.6 \%$ of the total collected isolates and $89 \%$ of $S$. aureus isolates which probably indicated the need for better understanding to infection control measures in the hospitals. Prevalence of MRSA in hospitals increased from 2.1\% in 1975 to 35\% in 1991 [24] and it was 46\% in Western Pacific region, 5.7\% in Canada, varied from less than $2 \%$ in the Netherlands to $54.4 \%$ in Portugal, from 23.6\% in Australia to more than $70 \%$ in Japan and Hong Kong [25] [26] and 30\% - 60\% in Thailand [27]. For serious MRSA infections, vancomycin is the treatment of choice and MRSA strains with reduced susceptibility to vancomycin have been reported [28] [29] but in this study no vancomycin resistant isolates were found. Only $10 \%$ of S. pneumonia isolates were resistant to penicillin which might indicate that the resistance to this bacteria in particular was not very high. However, this rate might increase if more awareness were not really considered at this stage. Hofmann et al. [30] reported that $25 \%$ of the isolates were resistant to penicillin. This may indicates that more resistant $S$. pneumonia isolates are on the way.

\section{Acknowledgements}

Thanks to all the staff members and the technicians of the Microbiology Lab., KAUH, Jeddah, Saudi Arabia.

\section{References}

[1] http://en.wikipedia.org/wiki/Jeddah

[2] Aarestrup, F.M., Wegener, H.C. and Collignon, P. (2008) Resistance in Bacteria of the Food Chain: Epidemiology and Control Strategies. Expert Review of Anti-Infective Therapy, 6, 733-750. http://dx.doi.org/10.1586/14787210.6.5.733

[3] Maragakis, L.L. and Perl, T.M. (2008) Acinetobacter baumannii: Epidemiology, Antimicrobial Resistance, and Treatment Options. Clinical Infectious Diseases, 46, 1254-1263. http://dx.doi.org/10.1086/529198

[4] Walsh, C.T. (2003) Antibiotics: Actions, Origins, Resistance. American Society for Microbiology, Washington DC. http://dx.doi.org/10.1128/9781555817886

[5] Levy, S.B. and Marshall, B. (2004) Antibacterial Resistance Worldwide: Causes, Challenges and Responses. Nature Medicine, S122-S129. http://dx.doi.org/10.1038/nm1145

[6] Atkinson, B.A. and Lorian, V. (1984) Antimicrobial Agent Susceptibility Patterns of Bacteria in Hospitals from 1971 to 1982. Journal of Clinical Microbiology, 20, 791-796.

[7] von Baum, H. and Marre, R. (2005) Antimicrobial Resistance of Escherichia coli and Therapeutic Implications. International Journal of Medical Microbiology, 295, 503-511. http://dx.doi.org/10.1016/j.ijmm.2005.07.002

[8] Kronvall, G.A. (2010) Antimicrobial Resistance 1979-2009 at Karolinska Hospital, Sweden: Normalized Resistance Interpretation during a 30-Year Follow-Up on Staphylococcus aureus and Escherichia coli Resistance Development. APMIS, 118, 621-639. http://dx.doi.org/10.1111/j.1600-0463.2010.02660.x

[9] Blaettler, L., Mertz, D., Frei, R., Elzi, L., Widmer, A.F., Battegay, M., et al. (2009) Secular Trend and Risk Factors for Antimicrobial Resistance in Escherichia coli Isolates in Switzerland 1997-2007. Infection, 37, 534-539. http://dx.doi.org/10.1007/s15010-009-8457-0

[10] Naas, T., Nordmann, P., Vedel, G. and Poyart, C. (2005) Plasmid-Mediated Carbapenem-Hydrolyzing Beta-Lactamase KPC in a Klebsiella pneumoniae Isolate from France. Antimicrobial Agents and Chemotherapy, 49, 4423-4424. http://dx.doi.org/10.1128/AAC.49.10.4423-4424.2005

[11] Schwaber, M.J. and Carmeli, Y. (2008) Carbapenem-Resistant Enterobacteriaceae: A Potential Threat. JAMA, 300, 2911-2913. http://dx.doi.org/10.1001/jama.2008.896

[12] Won, S.Y., Munoz-Price, L.S., Lolans, K., Hota, B., Weinstein, R.A. and Hayden, M.K. (2011) Emergence and Rapid Regional Spread of Klebsiella pneumonia Carbapenemase-Producing Enterobacteriaceae. Clinical Infectious Diseases, 53, 532-540. http://dx.doi.org/10.1093/cid/cir482

[13] Sanchez, G.V., Master, R.N., Clark, R.B., Fyyaz, M., Duvvuri, P., Ekta, G. and Bordon, J. (2013) Klebsiella pneumoniae Antimicrobial Drug Resistance, United States, 1998-2010. Emerging Infectious Diseases, 19, 133-136. http://dx.doi.org/10.3201/eid1901.120310

[14] Obritsch, M.D., Fish, D.N., MacLaren, R. and Jung, R. (2005) Nosocomial Infections Due to Multidrug-Resistant 
Pseudomonas aeruginosa: Epidemiology and Treatment Options. Pharmacotherapy, 25, 1353-1364

[15] Go, E.S., Urban, C., Burns, J., Kreiswirth, B., Eisner, W., Mariano, N., et al. (1994) Clinical and Molecular Epidemiology of Acinetobacter Infections Sensitive Only to Polymyxin B and Sulbactam. Lancet, 344, 1329-1332. http://dx.doi.org/10.1016/S0140-6736(94)90694-7

[16] Butler, M.S., Blaskovich, M.A. and Cooper, M.A. (2013) Antibiotics in the Clinical Pipeline in 2013. The Journal of Antibiotics, 66, 571-591. http://dx.doi.org/10.1038/ja.2013.86

[17] Wagenlehner, F.M., Weidner, W. and Naber, K.G. (2007) Pharmacokinetic Characteristics of Antimicrobials and Optimal Treatment of Urosepsis. Clinical Pharmacokinetics, 46, 291-305. http://dx.doi.org/10.2165/00003088-200746040-00003

[18] Livermore, D.M., Nichols, T., Lamagni, T.L., Potz, N., Reynolds, R. and Duckworth, G. (2003) Ciprofloxacin-Resistant Escherichia coli from Bacteraemias in England; Increasingly Prevalent and Mostly from Men. Journal of Antimicrobial Chemotherapy, 52, 1040-1042. http://dx.doi.org/10.1093/jac/dkg479

[19] Mavroidi, A., Miriagou, V., Liakopoulos, A., Tzelepi, E., Stefos, A., Dalekos, G.N. and Petinak, E. (2012) Ciprofloxacin-Resistant Escherichia coli in Central Greece: Mechanisms of Resistance and Molecular Identification. BMC Infectious Diseases, 12, 371. http://www.biomedcentral.com/1471-2334/12/371

[20] Manchanda, V., Sanchaita, S. and Singh, N.P. (2010) Multidrug Resistant Acinetobacter. Journal of Global Infectious Diseases, 2, 291-304. http://dx.doi.org/10.4103/0974-777X.68538

[21] Obritsch, M.D., Fish, D.N., MacLaren, R. and Jung, R. (2004) National Surveillance of Antimicrobial Resistance in Pseudomonas aeruginosa Isolates Obtained from Intensive Care Unit Patients from 1993 to 2002. Antimicrobial Agents and Chemotherapy, 48, 4606-4610. http://dx.doi.org/10.1128/AAC.48.12.4606-4610.2004

[22] Raja, N.S. and Singh, N.N. (2007) Antimicrobial Susceptibility Pattern of Clinical Isolates of Pseudomonas aeruginosa in a Tertiary Care Hospital. Journal of Microbiology, Immunology and Infection, 40, 45-49.

[23] Manno, G., Cruciani, M., Romano, L., Scapolan, S., Mentasti, M., Lorini, R., et al. (2005) Antimicrobial Use and Pseudomonas aeruginosa Susceptibility Profile in a Cystic Fibrosis Centre. International Journal of Antimicrobial Agents, 25, 193-197. http://dx.doi.org/10.1016/j.ijantimicag.2004.11.009

[24] Panlilio, A.L., Culver, D.H., Gaynes, R.P., Banerjee, S., Henderson, T.S., Tolson, J.S., et al. (1992) Methicillin Resistant Staphylococcus aureus in US Hospitals, 1975-1991. Infection Control and Hospital Epidemiology, 13, 582-586. http://dx.doi.org/10.2307/30148460

[25] Mekviwattanawong, S., Srifuengfung, S., Chokepaibulkit, K., Lohsiriwat, D. and Thamlikitkul, V. (2006) Epidemiology of Staphylococcus aureus Infections and the Prevalence of Infection Caused by Community Acquired Methicillin-Resistant Staphylococcus aureus in Hospitalized Patients at Siriraj Hospital. Journal of the Medical Association of Thailand, 89, S106-S117.

[26] Diekema, D.J., Pfaller, M.A., Schmitz, F.J., Smayevsky, J., Bell, J., Jones, R.N., et al. (2001) Survey of Infections Due to Staphylococcus Species: Frequency of Occurrence and Antimicrobial Susceptibility of Isolates Collected in the United States, Canada, Latin America, Europe, and the Western Pacific Region for the SENTRY Antimicrobial Surveillance Program, 1997-1999. Clinical Infectious Diseases, 32, S114-S132.

[27] Thamlikitkul, V., Jintanothaitavorn, D., Sathitmethakul, R., Vaithayaphichet, S., Trakulsomboon, S. and Danchaivijitr, S. (2001) Bacterial Infections in Hospitalized Patients in Thailand in 1997 and 2000. Journal of the Medical Association of Thailand, 84, 666-673.

[28] Hiramatsu, K., Hanaki, H., Ino, T., Yabuta, K., Oguri, T. and Tenover, F.C. (1997) Methicillin-Resistant Staphylococcus aureus Clinical Strain with Reduced Vancomycin Susceptibility. Journal of Antimicrobial Chemotherapy, 40, 135136. http://dx.doi.org/10.1093/jac/40.1.135

[29] Ploy, M.C., Grelaud, C., Martin, C., de Lumley, L. and Denis, F. (1998) First Clinical Isolate of Vancomycin-Intermediate Staphylococcus aureus in a French Hospital. Lancet, 351, 1212. http://dx.doi.org/10.1016/S0140-6736(05)79166-2

[30] Hofmann, J., Cetron, M.S., Farley, M.M., Baughman, W.S., Facklam, R.R., Elliott, J., Deaver, K.A. and Breiman, R.F. (1995) Prevalence of Drug-Resistant Streptococcus pneumoniae in Atlanta. The New England Journal of Medicine, 333, 481-486. http://dx.doi.org/10.1056/NEJM199508243330803 